\title{
4D MR Imaging Using Internal Respiratory Gating
}

\author{
M. von Siebenthal ${ }^{1}$, Ph. Cattin ${ }^{1}$, U. Gamper ${ }^{2}$, \\ A. Lomax $^{3}$, and G. Székely ${ }^{1}$ \\ 1 Computer Vision Laboratory, ETH, \\ CH-8092 Zürich, Switzerland \\ \{mvonsieb, cattin, szekely\}@vision.ee.ethz.ch \\ 2 Institute of Biomedical Engineering, ETH, \\ CH-8092 Zürich, Switzerland \\ 3 Paul Scherrer Institut, CH-5232 Villigen PSI, Switzerland
}

\begin{abstract}
Respiratory organ motion is a key problem in proton therapy and in many other treatments. This paper presents a novel retrospective gating method for 4D (dynamic 3D) MR imaging during free breathing to capture the full variability of respiratory organ deformation. In contrast to other imaging methods, a constant breathing depth or even strict periodicity are not assumed. 3D images of moving organs can be reconstructed for complete respiratory cycles by retrospective stacking of dynamic $2 \mathrm{D}$ images using internal image-based gating. Additional noise reduction by combining multiple images significantly increases the signalto-noise ratio. The resulting image quality is comparable to breath-hold acquisitions. Although the method was developed for proton therapy planning, the new possibilities to study respiratory motion are valuable to improve other treatments and to assess gating techniques, which rely on stronger assumptions about the breathing pattern.
\end{abstract}

\section{Introduction}

Respiratory organ motion is a complicating factor in proton therapy and other treatments. Especially for dynamic dose delivery, exact knowledge of organ motion and its influence on the dose distribution is crucial. The aim of the proposed method was to provide realistic 4D data (dynamic 3D images) of organ motion for the evaluation of dose delivery methodologies in proton therapy and for the assessment of external gating techniques. Existing 4D imaging methods are limited to strongly simplified breathing patterns or achieve only modest spatial or temporal resolutions. In this paper we present a novel method that can capture the full variability of organ motion. The method, which is applicable to any organ affected by respiratory motion, will be shown for the liver as an example.

The main component of liver motion is a cranio-caudal shift, usually in the range of $0.5-2.5 \mathrm{~cm}$ for quiet breathing [1]2]. As quantified in [2, the liver additionally shows motion in anterior-posterior $(1-12 \mathrm{~mm})$ and left-right direction $(1-3 \mathrm{~mm})$ as well as non-rigid deformations (up to $2 \mathrm{~cm}$ ). The breathing pattern 
may vary within short time scales in amplitude, frequency and shape. To capture this variability, we use MR Imaging. In contrast to $\mathrm{CT}$, volunteers are not exposed to ionizing radiation and the orientation of the scanned slices can be chosen freely. Nevertheless, some gating methods are common for MR Imaging and $\mathrm{CT}$, and therefore 4DCT is also considered in the following summary.

MR Imaging techniques are constantly evolving, but the required trade-off between resolution, acquisition speed and signal-to-noise ratio still prevents a detailed examination of organ motion in real-time. Dynamic 3D MR sequences like Fast Field Echo Planar Imaging (FFE-EPI) applied in 3 achieve high acquisition frequencies, but suffer from a reduced image quality and resolution if large regions like the lung or the liver are scanned. To overcome this trade-off, there are two main approaches, here denoted as breath-hold imaging and slice stacking. Breath-hold imaging uses static 3D volumes at different breathing depths determined for example with a stretch transducer [2] or a standard MR navigator 4 . One problem is that subjects may not be able to hold their breath for long periods like $40 \mathrm{~s}$ 4. Additionally, breath-hold images do not capture how the organ is deformed during motion. Even if the breath-hold positions lie in the range of free breathing, the hysteretic organ deformation between inhalation and exhalation cannot be captured 3 .

For slice stacking, one or a few 2D slices are scanned during a number of breathing cycles. Frames from these 2D sequences are retrospectively stacked to 3D images. The slice stacking methods found in the literature apply various gating methods, which use for example the tidal lung volume [5], infrared markers placed on the abdomen [67] or the abdominal skin detected in CT images [8]. In [7, an alternative internal gating method was proposed, which assumes a constant breathing amplitude.

To the author's knowledge, all gating methods that are currently used make strong assumptions on the regularity of the respiratory motion and parameterize this motion with a one-dimensional phase. Although respiration clearly shows a repetitive character, which is also exploited in this work, a reduction of the respiratory organ deformation to one parameter neglects all residual variability and may be a too coarse approximation in some cases. The purpose of the proposed imaging method is to overcome this issue and to reconstruct accurate $4 \mathrm{D}$ images from free-breathing sequences in an improved quality.

\section{Methods}

\subsection{Data Acquisition}

We propose an imaging method, which follows the slice stacking approach and captures the respiratory motion using free-breathing 2D MR images. In contrast to all techniques presented above, we use sagittal slices. This allows to track vascular structures during complete breathing cycles with minimal out-of-plane motion. Another advantage of this slice orientation is the reduction of blood flow artifacts in the liver region due to the beating of the heart. 
(a)

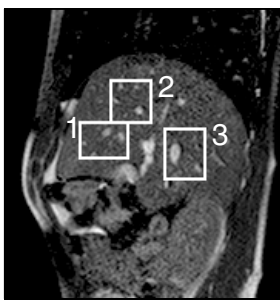

(b)

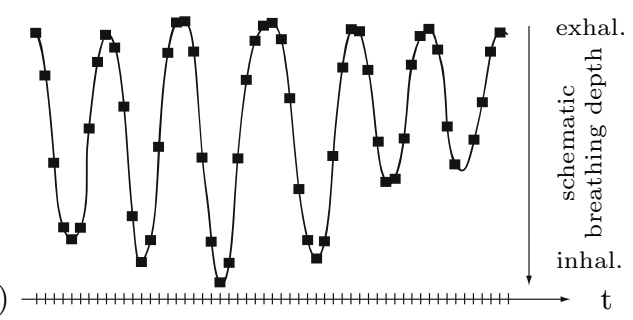

Fig. 1. (a) Navigator slice $N_{0}$. Regions 1-3 are considered for gating in Sect.2.2 (b) Schematic acquisition sequence.

To illustrate the $4 \mathrm{D}$ reconstruction, we start with a $2 \mathrm{D}$ image sequence of a dedicated slice called the navigator slice $N_{0}$ (Fig.11). Patient instruction ensures that this sequence captures a variety of different breathing depths. The aim is to reconstruct complete 3D images for all navigator frames in a complete breathing cycle. Therefore we need to acquire and identify fitting $2 \mathrm{D}$ images all across the liver. However, distant slices acquired during different breathing cycles are not directly comparable to the navigator slice. Different motion in distant slices can either reflect the relative motion in these regions or a change in the breathing pattern. To establish a relation between distant slices, we acquire pairs of slices, each pair containing the navigator slice, which is used for gating, and a data slice (Fig.21). The navigator slice stays at the same position for every pair $p$, whereas the position of the data slice is shifted across the liver. Each pair of slices is scanned for a certain time (Fig.2b). The data and navigator frames of pair $p$ acquired at times $t_{1}$ and $t_{2}$ are denoted $D_{p, t_{1}}$ and $N_{p, t_{2}} . N_{0, t}$ is the pure navigator sequence, which is acquired twice as fast as the pairs of slices.

The 2D images were acquired on a $1.5 \mathrm{~T}$ Philips Intera whole body MR system (Philips Medical Systems, Best, NL) with a Steady State Free Precession sequence, SENSE factor 1.7 and halfscan, 192x192 pixel and $1.8 \times 1.8 \mathrm{~mm}^{2}$ inplane resolution, flip angle $70^{\circ}, \mathrm{TR}=3.1 \mathrm{~ms}, 175 \mathrm{~ms}$ acquisition time per frame, with a coil array consisting of four rectangular elements. For pairs of slices, navigator and data frames were acquired alternately. At each of 30 slice positions we acquired 150 data frames and 150 navigator frames with $6 \mathrm{~mm}$ slice thickness and $3 \mathrm{~mm}$ overlap, yielding $3 \mathrm{~mm}$ through-plane resolution.

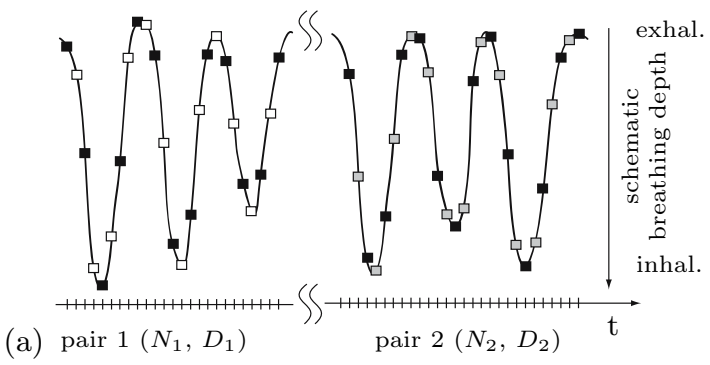

Fig. 2. Acquisition scheme. (a) Pairs of slices consisting of data slices $D_{p}$ and a common navigator slice $N_{p}$. (b) Schematic acquisition sequences for two pairs of slices. 


\subsection{Internal Gating}

To find matching 2D images, which can be stacked together to a 3D image, we propose an internal gating method based on the navigator images. This internal gating is less error-prone than external gating, which may fail to derive the accurate state of the organ from an external signal. To describe the proposed method, we consider a navigator frame $N_{0, i}$ acquired at time $i$, for which a matching data frame $D_{1}$ is searched. $N_{0, i-1}$ and $N_{0, i+1}$ are the neighboring navigator frames of $N_{0, i}$, whereas a candidate frame $D_{1, j}$ is embraced by the navigator frames $N_{1, j-1}$ and $N_{1, j+1}$ (Fig.(3). The proposed comparison is based on the assumption that the frames $N_{0, i}$ and $D_{1, j}$ show the same respiratory state, if the preceding navigator frames as well as the subsequent navigator frames are sufficiently similar. Thus, only navigator frames, which show the same slice of the liver, are compared to find fitting data frames. Therefore, different amplitudes or phase shifts at distant slices have no negative influence.

There are various possible similarity measures to compare the navigator frames. The evaluated options range from a simple sum of squared intensity differences to more sophisticated measures like the Mahalanobis distance in the eigenspace of the navigator frames. Since the goal is accurate slice fitting, a natural approach is to compare the position of prominent vascular structures and thus to directly quantify shift errors. This approach was chosen in the following and confirmed by the results. Figure 1a shows the three regions in the navigator frames that were considered in the presented example. The position of these regions was determined by template matching based on normalized cross correlation. Note that the apparent $2 \mathrm{D}$ translation detected by template matching may differ from the actual liver deformation, which in general has also an out-ofplane component. However, a similar apparent motion of all considered regions in both the preceding and the following navigator frames strongly indicates that the actual 3D deformation is similar as well.

To compare frames, we define a cost function $c(i, j)$, which should be small if the navigator frame $N_{0, i}$ and the data frame $D_{1, j}$ show the same respiratory state. Therefore, we require that each region $r$ is at a similar position in the two preceding navigator frames $N_{0, i-1}, N_{1, j-1}$ and in the subsequent navigator

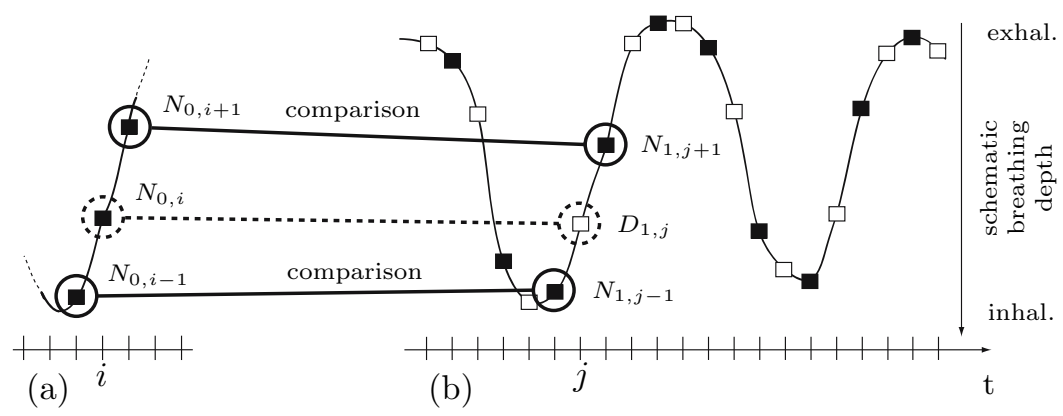

Fig. 3. (a) Navigator frame $N_{0, i}$, for which a matching data slice $D_{1, j}$ is searched in sequence (b) by comparing the neighboring navigator frames 
frames $N_{0, i+1}, N_{1, j+1}$. The following cost function sums up the vectorially added deviations of the considered regions in the preceding navigator frames $\Delta \boldsymbol{X}_{-1}^{r}$ and the deviations in the subsequent navigator frames $\Delta \boldsymbol{X}_{+1}^{r}$. With coordinate axes in anterior-posterior and cranio-caudal direction, $x_{i}^{r}$ and $y_{i}^{r}$ are the coordinates of region $r$ at time $i$. With $P$ the number of tracked regions we define

$c(i, j)=\sum_{r=1}^{P}\left\|\Delta \boldsymbol{X}_{-1}^{r}+\Delta \boldsymbol{X}_{+1}^{r}\right\|=\sum_{r=1}^{P}\left\|\left(\begin{array}{c}x_{j-1}^{r}-x_{i-1}^{r} \\ y_{j-1}^{r}-y_{i-1}^{r}\end{array}\right)+\left(\begin{array}{c}x_{j+1}^{r}-x_{i+1}^{r} \\ y_{j+1}^{r}-y_{i+1}^{r}\end{array}\right)\right\|$.

Compared to the simple Euclidean distance, the vectorial displacements capture the actual motion more consistently and proved to be more robust. The best matching data frame $D_{1, j^{*}}$ for a given navigator frame $N_{0, i}$ is found at time

$$
j^{*}=\arg \min _{j} c(i, j) .
$$

For the navigator frame $N_{0, i}$, the best matching data frames from each pair of slices $D_{p, j^{*}}, p=1,2,3, \ldots$, are combined to a 3D stack. Such a stack is reconstructed for every navigator frame $N_{0, i}, N_{0, i+1}, N_{0, i+2}, \ldots$ in an entire breathing cycle to obtain a complete $4 \mathrm{D}$ data set with the temporal resolution of the pure navigator sequence $N_{0}(5.7 \mathrm{~Hz})$.

\subsection{Noise Reduction}

The acquired images show a considerable amount of noise due to their short acquisition time and high spatial resolution. To improve the 4D reconstruction, a straight-forward noise reduction was applied. The developed gating method was used to find not only one but several frames showing possibly similar respiratory states. These frames were averaged, which artificially prolonged the acquisition time of the resulting image. Assuming additive noise, an averaging over $M$ frames reduces the variance of the noise by a factor of $M$. The number of frames that can be averaged is limited by the duration of the acquisition session. In the presented example, the acquisition time was $30 \mathrm{~min}$ and five frames were averaged.

\section{Results and Evaluation}

The proposed imaging method was performed for six healthy volunteers. A 4D data set of a male volunteer is used to illustrate the method in the following. For comparison, Fig. 四 shows three cuts through a breath-hold image close to relaxed exhalation. A 3D image reconstructed from 30 free-breathing images using the proposed method is shown in Fig.4 4 . The blood vessels and liver boundaries exhibit no major discontinuities. Note that Fig.4b shows the reconstructed 3D image that is most similar to the breath-hold image. However, the free-breathing image looks different from the breath-hold image, because the latter cannot capture the shape of the moving organ, which undergoes a hysteretic deformation between inhalation and exhalation. Figure 4 r shows the same free-breathing 
(a)

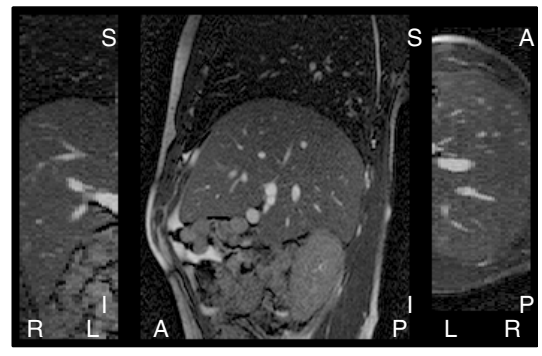

(a) Breath-hold image.

(b) Reconstruction from free-breathing $2 \mathrm{D}$ images.

(c) Reconstruction from free-breathing $2 \mathrm{D}$ images with noise reduction. Average over five images.

(b)

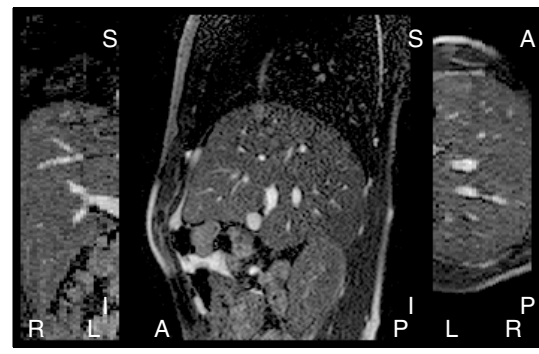

(c)

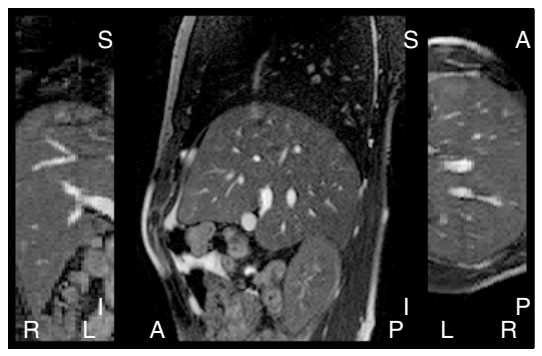

Fig. 4. Orthogonal cuts through $3 \mathrm{D}$ images of the right liver lobe

image with additional noise reduction as discussed in Sect.2.3. The signal-tonoise ratio is remarkably improved and the resulting images achieve the quality of breath-hold acquisitions. The averaging of several images did not introduce significant blurring, which indicates that the number of acquisitions at each position was sufficient and enough similar frames were found. The darkband artifact through the dome of the liver can be shifted by proper shim adjustment.

In addition to the visual assessment of the reconstructed data sets, which showed well fitting 3D images, a rigorous validation is desirable but not straightforward. However, the proposed frame matching algorithm can be tested experimentally to evaluate the plausibility of the $4 \mathrm{D}$ data. To test if matching data frames are found, a leave-one-out experiment was performed. A sequence of navigator frames $N_{1}$ and data frames $D_{1}$ (288 frames in total) at $3 \mathrm{~cm}$ distance was considered. For each data frame $D_{1, i}$ in this sequence, the best matching data frame $D_{1, j}$ was searched among the remaining frames $(i \neq j)$ according to the cost function $c(i, j)$ defined in Eq. (11). The similarity of the selected data frame $D_{1, j^{*}}$ to the "ideal" left-out frame $D_{1, i}$ was quantified as the deviation of four selected regions within $D_{1}$ (Fig.5) determined by template matching based on normalized cross correlation. While the respiratory motion ranged up to $11 \mathrm{~mm}$ in this example, the resulting mean deviations of the considered regions were in the range of $0.3-0.4 \mathrm{~mm}$, which is less than half a voxel $(0.9 \mathrm{~mm})$.

\section{Discussion and Outlook}

The developed technique allows for the reconstruction of $4 \mathrm{D}$ data sets showing the detailed deformation of an organ during free breathing including the 
(a)

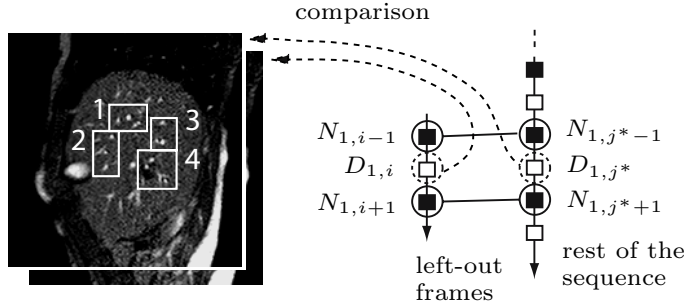

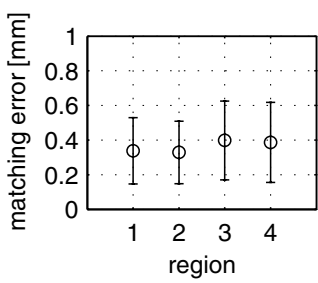

Fig. 5. Leave-one-out experiment. (a) The data frame $D_{1, j^{*}}$ (found by navigator comparison) is compared to the "ideal" left-out frame $D_{1, i}$. (b) Mean error and its standard deviation for four regions.

hysteretic deformation between inhalation and exhalation. As opposed to other $4 \mathrm{D}$ imaging techniques, no strict assumptions on the regularity of the respiratory motion such as constant breathing depth or even periodicity are made. This allows to better study the intra-subject as well as the inter-subject variability of respiratory motion and to analyze these variations statistically. Provided that a sufficient amount of raw data was acquired, 4D data sets can be reconstructed for arbitrary amplitudes, frequencies and shapes of the breathing pattern.

Evaluation experiments have shown that the proposed gating method accurately finds fitting $2 \mathrm{D}$ images with minimal deviations based on the dedicated navigator slice, even if the considered slices are several centimeters apart. A gating scheme with overlapping pairs of neighboring slices as an alternative but would likely lead to error propagation from one overlapping pair to the next one.

For a rigorous validation of the proposed method, either experiments with a realistically deformable phantom or an in silico simulation of the entire data acquisition chain are possible. The second option will be investigated in the future work, for example to determine the maximum viable distance between a data slice and the navigator slice.

A common limitation for all slice stacking methods is that only breathing cycles can be reconstructed, for which all necessary frames were captured across the liver. If particularly deep breathing is only acquired at some of the slice positions, the method fails to reconstruct a complete $4 \mathrm{D}$ data set. This issue was addressed by instructing the volunteers to intentionally breath deeply during parts of each acquisition, which ensured that a variety of breathing depths was captured at each slice position.

Another observed issue was the drift of the exhalation position during the acquisition of most data sets. This drift ranged up to $7 \mathrm{~mm}$ in one case. On the one hand, this issue is aggravated by the long total acquisition time of half an hour in our studies. But on the other hand, the problem of variation beyond a regular breathing cycle is simply ignored by other 4D imaging approaches and can now be addressed specifically with the proposed method. Drift and other irregularities are recognized and handled by the internal gating technique. One possibility to alleviate the impact of organ drift is to change the acquisition sequence. The applied procedure selects a slice and completely captures its dynamic behavior 
before moving to the next spatial position. An interleaved sequence, which goes back and forth through all slices, would increase the probability that we acquire sufficient data for a complete $4 \mathrm{D}$ reconstruction before a major drift occurs.

Despite the discussed limitations, the obtained results demonstrate the potential of the proposed method for $4 \mathrm{D}$ imaging in surpassing quality. The technique is applicable to any organ that undergoes respiratory motion like the lung or the kidneys and can be implemented using a standard MR scanner without additional equipment. Although the proposed method was developed for proton therapy planning, the new possibilities to study realistic respiratory motion are valuable to improve many other techniques, first of all conventional radiation therapy planning. Other particularly interesting applications are the evaluation and the comparison of gating techniques that inherently rely on stronger assumptions about the respiratory motion. Furthermore, generic motion data, which is not patient specific, is useful for the evaluation of dose delivery methodologies and anatomical simulations or for advanced applications like model-based segmentation or tracking. Numerous research projects in these fields may profit from a more accurate and more reliable $4 \mathrm{D}$ imaging using the proposed method.

Acknowledgments. This work has been supported by the CO-ME/NCCR research network of the Swiss National Science Foundation (http://co-me.ch).

\section{References}

1. Davies, S., Hill, A., Holmes, R., et al.: Ultrasound quantitation of respiratory organ motion in the upper abdomen. Br. J. Radiol. 67 (1994) 1096-1102

2. Rohlfing, T., Maurer, C., Jr., O'Dell, W., Zhong, J.: Modeling liver motion and deformation during the respiratory cycle using intensity-based free-form registration of gated MR images. Proc. SPIE Med. Imaging 20014319 (2001) 337-348

3. Blackall, J., Ahmad, S., Miquel, M., et al.: Modelling respiratory motion for optimisation of lung cancer radiotherapy using fast MR imaging and intensity-based image registration. In: Proc. Intl. Soc. Mag. Reson. Med. (2004) 12:2610

4. Blackall, J.: Respiratory Motion in Image-Guided Interventions of the Liver. PhD thesis, University of London (2002)

5. Low, D., Nystrom, M., Kalinin, E., Parikh, P., et al.: A method for the reconstruction of four-dimensional synchronized CT scans acquired during free breathing. Med. Phys. 30 (2003) 1254-1263

6. Ford, E., Mageras, G., Yorke, E., Ling, C.: Respiration-correlated spiral CT: A method of measuring respiratory-induced anatomic motion for radiation treatment planning. Med. Phys. 30 (2003) 88-97

7. Pan, T., Lee, T., Rietzel, E., Chen, G.: 4D-CT imaging of a volume influenced by respiratory motion on multi-slice CT. Med. Phys. 31 (2004) 333-340

8. McClelland, J., Chandler, A., Blackall, J., et al.: Feasibility study of a novel technique for constructing respiratory motion models, for use in $4 \mathrm{D}$ lung cancer radiotherapy planning. In: Proc. MIUA. (2004) 220-3 